\title{
A review of childhood vestibular disorders
}

\section{Rogers MSc(Aud)}

To cite this article: C Rogers MSc(Aud) (2010) A review of childhood vestibular disorders, South African Family Practice, 52:6, 514-517, DOI: 10.1080/20786204.2010.10874036

To link to this article: http://dx.doi.org/10.1080/20786204.2010.10874036
6 (c) 2010 SAAFP. Published by Medpharm.

\section{Published online: 15 Aug 2014.}

Submit your article to this journal $\widetilde{\lambda}$

Џll Article views: 397

Q View related articles $\widetilde{ }$ 


\title{
A review of childhood vestibular disorders
}

\author{
Rogers C, MSc(Aud) \\ Division of Communication Sciences and Disorders, School of Health and Rehabilitation Sciences, \\ Faculty of Health Sciences, University of Cape Town \\ Correspondence to: Christine Rogers, e-mail: Christine.Rogers@uct.ac.za \\ Keywords: dizziness; paediatric; vertigo; vestibular disorders
}

\begin{abstract}
Diagnosis of disorders of balance is challenging, as there are broad differentials and patients may present to a variety of healthcare practitioners, ranging from an audiologist to a psychiatrist. In addition, investigations, both at the bedside and laboratory, are often non-contributory, expensive and unpleasant. The adult dizzy patient is regarded with dread by many in the healthcare community: the patient is difficult to diagnose and challenging to treat, and the situation is frequently complicated by the presence of anxiety, panic and depression. When symptoms arise in childhood they cause alarm in the parents and the treating healthcare professional. Diagnosis and management of balance disorders in childhood, is even more demanding when patients are frequently unable to communicate the nature of the complaint. Furthermore, the aetiology and presentation of vestibular disturbance is markedly different between adults and children. Symptoms of vestibular disorders in children may easily be mistaken for behavioural or other medical problems, leading to underdiagnosis and inappropriate investigations and treatment. Detrimental effects of childhood vestibular disorders may include delayed gross motor development, learning and spatial problems, and time off school. This review summarises the most common causes of childhood vestibular disorders and suggests the need for a multidisciplinary approach to assessment.
\end{abstract}

(P) Peer reviewed. (Submitted: 2009-12-23, Accepted: 2010-02-06). ๑ SAAFP

SA Fam Pract 2010;52(6):514-517

\section{Introduction}

The sense of balance and postural control is underpinned by three main inputs: the vestibular contribution from the labyrinth and connecting peripheral and central pathways, visual inputs which aid orientation, and the sense of proprioception. In addition, the areas within the brainstem that are responsible for integrating the three received signals, and producing motor outputs in terms of reflexive instructions to the eyes and spine, all need to be fully functional. In the face of unexpected perturbations of balance, decisions regarding postural control are made on an instantaneous basis without any conscious input from the higher centres in the brain. When disease causes a breakdown of incoming signals from within or between any of the three inputs, or a failure to integrate them successfully, the subjective complaint registers as either symptoms of dizziness or vertigo. Dizziness is an all-encompassing term often used by patients to describe any altered sensation of orientation to the environment and includes presyncope, light-headedness and ataxia, all of which may have a variety of aetiologies. Vertigo, which may be defined as a hallucination of movement, ${ }^{1,2}$ usually suggests vestibular involvement. Even after a catastrophic loss of one of the balance organs, which could potentially be life threatening, the brain has built-in compensatory mechanisms that reduce the perception of error signals, which in turn eases the associated nystagmus, nausea and vomiting, and avoids electrolyte imbalance and possible death. This process of vestibular compensation is triggered immediately after a unilateral, acute vestibular insult, and within days patients have achieved control over symptoms with the head static. However, control of the mismatch of incoming signals in a dynamic situation, such as when the head or head and body are moving, takes longer. It is thought that children may recover so quickly that the presence of an acute vestibular crisis may be masked. ${ }^{2}$

Diagnosis and management of vestibular disorders in childhood is demanding for a variety of reasons. First, vestibular or balance disorders are often perceived to be a problem that only affects adults. However, although unusual, they do exist in children. Furthermore, the aetiologies and pathologies that affect children and result in balance problems are quite different to those that may be responsible in the case of the vertiginous adult. For example, benign paroxysmal positional vertigo (BPPV), a mechanical disorder of the otoliths, is the most common cause of vertigo in adults, but is extremely rare in children. $A$ potential precursor of migraine, benign paroxysmal vertigo of childhood (BPVC), is unique to the paediatric population and is one of the most frequently occurring childhood vestibular disorders. Second, there is little focus on vestibular disorders of childhood during the training of many healthcare practitioners. In addition, due to the marked differences in the presentation of vestibular disorders in childhood, complaints of dizziness may not necessarily be linked to a cause within the vestibular system, resulting 
in under-referral to specialities that may be able to assist, such as ENT surgeons or neurologists. Third, while adults are able to communicate descriptions of altered balance, children present special challenges. Finally, the subjective complaints of dizziness and vertigo are often not amenable to assessment in the test laboratory and may be refractory to treat. Expensive and potentially distressing investigations such as MRI or specialised vestibular testing frequently do not contribute to either the diagnosis or therapy plans. Moreover, even if the tests suggest pathology, they do not give any indication of the patient's functional capacity, which is dependent on his or her ability to compensate for the effect of vestibular lesions. ${ }^{1,3}$

This review presents current knowledge about the clinical approach to the diagnosis and management of common childhood vestibular disorders. Symptoms of these disorders, a brief review of the tests that may be helpful, and treatment of the most common pathologies will be discussed.

An extensive case history should include focused questions about the precise quality of the symptoms, duration and onset, and associated features of the attack, developmental milestones and a full medical and neuro-otological history, including a history of migraine in the family. The case history can contribute significantly to diagnosis in the majority of cases of dizziness and vertigo. ${ }^{4}$ However, the usefulness of the case history depends on both the patient's ability to convey the nature of the complaint as well as the clinician's in-depth knowledge of possible aetiologies.

\section{Symptoms of childhood vestibular disorders}

Dizziness as a symptom is non-specific. However, the description of vertigo may narrow down the aetiology as it suggests vestibular involvement, which in most cases is due to a unilateral lesion. When vertigo is caused by a sudden unilateral vestibular hypofunction, for example, vestibular neuritis, it may be so severe that it is incapacitating. It should be noted that both dizziness and vertigo are symptoms, not diagnoses. ${ }^{2}$ Due to the novel nature of the symptoms, often even adults have difficulty in describing the sensations they experience. Children may not have the vocabulary to give an accurate account of symptoms, so distinguishing between dizziness and vertigo, and thus refining differential diagnosis, is difficult. As is to be expected, these are frightening symptoms for the child and parents. In older children, it may be helpful to ask the child to compare the symptoms to experiences such as on merrygo-rounds in order to establish if an illusion of movement is present (i.e. true vertigo). ${ }^{4}$ In children who are too young to describe their symptoms, the clinician must rely on the parents' description. In cases of recurrent episodes, it could be very helpful to have attacks videotaped and brought to the consultation, as signs may be absent should the child be seen between episodes.

Figure 1, adapted from Mehta and Stakiw, ${ }^{5}$ summarises commonly reported symptoms associated with acute childhood vestibular disorders. It should be noted that symptoms can often be mistaken for behavioural issues or simple clumsiness. ${ }^{4}$ Children with congenital hearing loss may present with loss of postural control and delayed gross motor milestones. Fine motor skills are usually normal and children do catch up gross motor skills later due to the plasticity of the developing nervous system. ${ }^{6}$ However, when learning to walk, falls may be much more frequent.

\section{Evaluation of the dizzy child}

Ideally, an evaluation of the vestibular system in the young child should be performed in conjunction with a paediatrician, ENT specialist or neuro-otologist, and must include assessment of muscle tone, head and postural

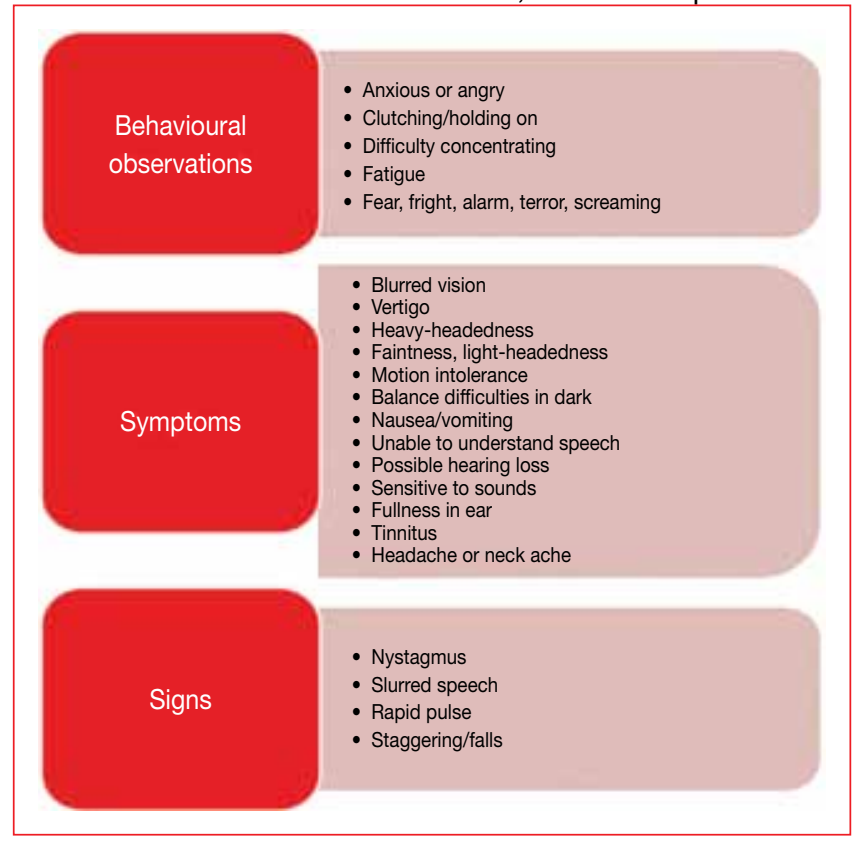

Figure 1: Behavioural observations, signs and symptoms of acute episodes of childhood vestibular disorders

control, and developmental reflexes. It may be necessary to involve other disciplines, such as audiologists and physiotherapists. As with the vertiginous adult, children should have an audiologic assessment. Evaluation of hearing may be challenging in small children and the audiologist will select the best method (either behavioural or electrophysiologic) to obtain accurate results. Establishing the presence and aetiology of any hearing loss is extremely helpful. For example, should otitis media with effusion (OME) be established, any further investigation of the imbalance may be deferred until the OME has resolved, as frequently balance problems are transient with $\mathrm{OME}{ }^{7}$ The role of the physiotherapist is highlighted when evaluation of fine and gross motor functions is required, for example in the case of congenital hearing loss. Both physiotherapists and specially trained audiologists may also be involved with vestibular rehabilitation therapy programmes that may be indicated especially for the child with congenital hearing loss. For the physical examination, some of the tests used in adult clinical assessment are equally helpful in children. Tests can be made into fun games and, therefore, accomplished relatively easily with cooperative preschoolers. Tests of dorsal column function include the Romberg and sharpened Romberg (standing with the feet in a tandem position with 
the eyes open and then closed). The headshake (called head-motion in the paediatric setting), dynamic visual acuity and post-rotatory tests are all simple tests of the vestibulo-ocular reflex pathways which can be administered by GPs and specialists alike. The head-motion test and post-rotatory tests will detect asymmetries in vestibular function, while the dynamic visual acuity test is more likely to detect bilateral vestibular hypofunction as it examines the ability to maintain an image on the fovea during static and head-moving conditions. The tests vary in sensitivity and specificity, but the combined results from the clinical examination are useful in identifying which children may need further referral. It is most likely that the patient will be seen when he or she is not having an acute attack; however, the vestibulo-ocular reflex pathway testing described will detect residual unilateral hypofunction. Laboratory testing includes electro- or video-nystagmography, the mainstay of which is caloric irrigations, rotary chair, posturography and vestibular myogenic potentials (VEMPs). Formal laboratory vestibular and other tests, such as blood cell counts and imaging, should only be undertaken purposefully, to confirm a diagnosis and plan of action. ${ }^{8}$ It should be noted that not every dizzy patient requires sophisticated, equipmentbased vestibular testing, and a policy of requesting extensive investigations for all patients with dizziness and vertigo should be avoided. ${ }^{8}$

\section{Common childhood disorders}

A search of the literature suggests that there appears to be no incidence data for either adult or childhood vestibular disorders in Africa. A study in India focused only on adults, ${ }^{9}$ and appears to be the only large-scale study of the prevalence of vestibular disorders in a developing country.

\section{Migrainous equivalents}

Migraine equivalent is the most frequent diagnosis for children with vertigo residing in developed countries (25\%). ${ }^{1,2}$ In contrast to migraine with headache in adults, other presentations of migraine, including recurrent vertigo and dysequilibrium, are more frequent in children. Episodic vertigo is associated with headaches. However, the headache may not necessarily have a temporal relationship to the vertigo. Features of the attack include nausea, vomiting and photophobia, and result in distressed behaviour. Duration can be several hours and episodes are more likely to occur when the child is tired. Otological, vestibular and neurologic investigations are all normal. Due to the association of migraine equivalents with eye problems, patients should consult an ophthalmologist to exclude refraction problems or disorders of ocular convergence. ${ }^{1}$ Diagnosis of migrainerelated dizziness remains a process of exclusion; first, other causes of recurrent episodic vertigo, such as Ménière's disease and some forms of seizure disorders, have to be ruled out. $^{2}$

\section{Benign paroxysmal vertigo of childhood}

The cause of BPVC is thought to be due to an interuption of blood supply to the brain. ${ }^{5}$ BPVC is the second most common cause of vertigo in children (20\%), especially in small children between 2 and 3 years of age. ${ }^{1}$ Attacks begin between the ages of 1 and 4 years, but can occur below the age of 12 months or up to the age of 10 years. ${ }^{5}$ However, should onset occur in the older child (> 4-5 years), rigorous attempts should be made to exclude other causes, including opthalmological, as mentioned above. ${ }^{1}$ Recurrent, spontaneous episodes of true vertigo, nystagmus, ataxia and dysequilibrium occur with a duration usually less than 10 minutes, although they can last hours. ${ }^{1,3,5,10}$ During the attack, although it is frightening, consciousness is not affected and the child is able to remember the episode. Behavioural responses to a severe attack range from the child keeping completely still and being unable to move, to becoming limp. Children may hold on to a stationary object or parent. Once the episode has passed, the child is able to return to normal activities immediately. The frequency of attacks may range from weekly to every six months. There are no obvious triggers for the attacks, such as positioning changes. Children with BPVC will not have hearing loss or tinnitus and are otherwise healthy. Headache is not a major symptom, but between 30 and $50 \%$ of children with BPVC will develop typical migraine later. Most children grow out of the condition by about the age of $8 .^{1,2,5,10}$

\section{Otitis media with effusion}

Otitis media can be regarded as a continuum, through acute, in which signs and symptoms of acute inflammation are found, to OME, a relatively silent condition in which serous fluid may be present in the middle ear for weeks or months. ${ }^{7}$ While parents often report increased balance problems and clumsiness in their children with OME, the precise manner in which OME causes balance dysfunction is still unclear. Due to thin membranes separating the middle and inner ears, it is possible that changes in air pressure and the transfer of toxins via the round window may cause pathological changes in the inner ear and subsequently result in balance difficulties. $^{2,7}$

There is a suggestion that children with OME are more reliant on visual rather than vestibular and proprioceptive inputs for maintaining postural control. This increased visual dependence resembles the behaviour and adaptive strategies of adults who have vestibular deficits, and could imply that either vestibular information is reduced in magnitude or not used optimally. Therefore, in children who are visually dependent, play that jumbles the visual environment, such as jumping, skipping and using playground equipment, like jumping castles and merry-gorounds, could promote a sensory mismatch which, in turn, could make the child clumsy and more prone to accidents or falls. ${ }^{7}$

\section{Management}

\section{Migraine equivalents}

Management strategies include identification of triggers, including ophthalmological problems, sleep quality and stressors. Simple analgesics can be used, either alone or with anti-inflammatories. This management is often adequate and may reduce the number of attacks as well as their severity. It is suggested that, should these measures fail, the child should be referred to a specialist 
for further assessment and management. For example, more specific treatments, such as the use of prophylactics, may be considered. However, some drugs, such as antiserotoninergic derivatives, are not suitable for children under the age of 12 years. ${ }^{1}$

\section{Benign paroxysmal vertigo of childhood}

Parental counselling and support is very important, as the attacks are frightening to witness if severe. The benign and self-limiting nature of the condition should be emphasised. In general, no treatment is necessary but the child will need comforting during the attacks. MRI is not helpful if the other examinations (e.g. clinical, neurological and audiological) are normal, and often presents a risk for the small child.

\section{Otitis media}

Treatment should follow the usual strategies and be completed before a decision is made as to whether to investigate the vestibular aspects further. It should be noted that bilateral otitis media has a more marked effect on balance than unilateral otitis media, and that most of the balance problems experienced resolve with the placement of grommets. ${ }^{7}$

\section{Conclusion}

Childhood vestibular disorders may be more common than traditionally believed. Three causes of dizziness and vertigo have been discussed. Young children are often not able to describe their symptoms, leading to mis- and underdiagnosis. An example of this is migraine, which is often thought to only affect adults. It presents in more unusual ways in children, and can present as vestibular disturbance without headache. Assessment and management is best performed by a team of professionals and the caregivers. The presence of hearing loss is an important indicator when determining the direction of further investigations in all dizzy patients. It is argued that establishing auditory function may be a helpful and often overlooked step in terms of differential diagnosis and so the audiologist may play a valuable role. In addition, some audiologists have advanced training in vestibular assessment and management and, together with physiotherapists, are able to offer rehabilitative services. The primary care physician plays an important role in the care of the dizzy child. First, he or she will be able to administer bedside tests to identify any possible vestibular hypofunction, which should then be referred to the ENT surgeon. Second, OME is a common disease of childhood and the primary care practitioner is ideally placed to either rule this out or to provide first-line treatment. Cases that fail to settle may need to be referred to the ENT specialist for tympanostomy tube insertion. Hence, the ENT specialist is another key member of the multidisciplinary care team. Should a migraine equivalent or BPVC be suspected, counselling and treatment can be provided. If baseline treatment fails, the GP can refer the patient to a neurologist. Attacks of vertigo may be poorly tolerated and upsetting for children, thus management of this aspect is important, and both the child and parents need to be counselled. Children with vestibular disorders may need additional assistance at school, as they may face challenges with learning and spatial concepts as well as some physical activities, such as riding bicycles or skateboards. Days at school may be lost due to migraine, which then adds to the pressure of keeping up in class. Vestibular disorders may be disruptive for the whole family, especially if it is a chronic condition, so support should be given wherever possible.

Statement of conflict of interest: I declare that I have no financial or personal relationship which may have inappropriately influenced me in writing this paper.

\section{References}

1. Wiener-Vacher S. Vestibular disorders in children. Int $\mathrm{J}$ Audiol 2008;47(9):578-83.

2. Casselbrant M, Mandel, E. Balance disorders in children. Neurol Clin 2005;23(3):807-29.

3. Worden B, Blevins N. Pediatric vestibulopathy and pseudo-vestibulopathy: differential diagnosis and management. Curr Opin Otolaryngol Head Neck Surg 2007;15(5):304-9.

4. Nandi R, Luxon, L. Development and assessment of the vestibular system. Int J Audiol 2008;47(9):566-77.

5. Mehta Z, Stakiw, D. Childhood vestibular disorders: a tutorial. Comm Disord Q 2004;26(1):5-16.

6. Kaga $\mathrm{K}$, Shinjo $\mathrm{Y}$, Jin $\mathrm{Y}$, Takegoshi $\mathrm{H}$. Vestibular failure in children with congenital deafness. Int J Audiol 2008;47(9):590-9.

7. Casselbrant M, Villardo R, Mandel E. Balance and otitis media with effusion. Int J Audiol 2008;47(9):584-9.

8. Ravid S, Bienkowski, R, Eviatar, L. A simplified diagnostic approach to dizziness in children. Pediatr Neurol 2003;29(4):317-20.

9. Abrol R, Nehru V, Venkatramana Y. Prevalence and etiology of vertigo in adult rural population. Indian J Otolaryngol Head Neck Surg 2001;53(10):32-6.

10. Hubbell R, Skoner, J. Vertigo in children. Curr Opin Otolaryngol Head Neck Surg 1999;7(6):328-34. 